\title{
Posturas didácticas sobre el método científico y desarrollo de competencias investigativas en estudiantes universitarios
}

\author{
Didactic positions on the scientific method and development of research skills in \\ university students
}

\author{
Héctor Raúl Zacarías Ventura1,a \\ https://orcid.org/0000-0002-7210-5675 \\ Jesús Vilchez Guizado 2,b \\ https://orcid.org/0000-0001-9941-2563 \\ José Antonio Supo Condori ${ }^{3, c}$ \\ https://orcid.org/0000-0002-7115-4563
}

\section{Citar como}

Zacarías, H., Vilchez, J. y Supo, J. (2021). Posturas didácticas sobre el método científico y su influencia en el desarrollo de competencias investigativas en estudiantes universitarios. Desafíos, 12(2); 09-14. https://doi.org/10.37711/desafios.2021.12.2.341

\section{RESUMEN}

Objetivo. Evidenciar que las posturas didácticas con enfoque cuantitativo vs. conceptualista-epistemológico del docente sobre el método científico influyen en el desarrollo de competencias investigativas en el estudiante universitario. Métodos. Consistió en una investigación pura o básica, de tipo observacional, analítico, prospectivo y transversal. El nivel de la investigación fue explicativo, evaluó la causalidad de una variable sobre la otra. La población estuvo conformada por 570 estudiantes del último ciclo de estudios de la Universidad de Huánuco, matriculados en el ciclo académico 2020-I. La muestra estuvo conformada por 215 estudiantes, elegidos mediante un muestreo aleatorio estratificado. Se empleó un instrumento de medición documental adaptado de la escala de evaluación de competencias investigativas de Jaik y Ortega (2017). Resultados. Los resultados confirman que la postura didáctica del docente sobre el método científico influye en las competencias investigativas del estudiante universitario, con una probabilidad de error del 3,2 \%. Conclusiones. Se concluye que la postura didáctica del docente sobre el método científico efectivamente influye en el desarrollo de competencias investigativas del estudiante universitario.

Palabrasclave:metodología;trabajodeinvestigación;formaciónprofesional;enfoquecientífico;investigaciónbásica.

\section{ABSTRACT}

Objective. To show that the didactic positions with quantitative vs. conceptualist-epistemological approach of the teacher on the scientific method influence the development of research skills in the university student. Methods. It consisted of pure / basic, observational, analytical, prospective and transversal research. The level of research was explanatory, assessing the causality of one variable over the other. The population consisted of 570 students from the last semester of studies of Universidad de Huánuco, enrolled in the academic cycle 2020-I. The sample consisted of 215 students, chosen by means of a stratified random sample. A documentary measurement instrument adapted from Jaik and Ortega's scale of assessment of investigative competencies (2017) was used. Results. The results confirm that the didactic posture of the teacher on the scientific method influences the research skills of the university student, with a probability of error of $3.2 \%$. Conclusions. It is concluded that the didactic posture of the teacher on the scientific method effectively influences the development of research skills of the university student.

Keywords: methodology; research work; professional training, scientific approach; basic research.

\section{Filiación y grado académico}

1 Universidad de Huánuco, Huánuco, Perú.

2 Universidad Nacional Hermilio Valdizán, Huánuco, Perú.

${ }^{3}$ Sociedad Hispana de Investigadores Científicos, Arequipa, Perú. a Magister en Docencia en Educación Superior e Investigación.

b Doctor en Educación.

Doctor en Salud Pública. 


\section{INTRODUCCIÓN}

La educación superior universitaria tiene entre sus fines impulsar la productividad científica, lo que incluye la producción de aquellos conocimientos que se distinguen del conocimiento vulgar debido a que, para su creación, se ha seguido el método científico, lo cual permite catalogarlos además como un conocimiento refinado. La producción científica es un quehacer integrado por numerosas y diversas operaciones orientadas a un propósito común; por tal razón, la enseñanza de estas operaciones amerita tanto del conocimiento de un especialista como del trabajo de un relojero para poder articular lo diverso con lo particular de las tareas involucradas en aquel (Sánchez, 2014). Ampliando el razonamiento, Salazar (2004) refiere que la formación del estudiante en la actividad científico-investigativa es definitivamente una responsabilidad interdisciplinaria. Abreu et al. (2017) señalan además que la didáctica es una de las ciencias de la educación en pleno y constante desarrollo, y está estrechamente relacionada con otras ciencias que actúan en el proceso de enseñanza-aprendizaje integrado e institucionalizándolo, especialmente con la pedagogía, pero también mantiene sus propias particularidades y su esencia.

El desarrollo de las competencias investigativas en los estudiantes universitarios involucra el compromiso sincero de los docentes con la actividad (Arbeláez et al., 2006). Orozco (2013) refiere que la metodología para realizar tesis tiene pilares sobre los que se construye, que incluyen, en primer lugar: los principios filosóficos aplicados al método científico, la concepción científica del mundo del investigador y su ideología. En la vida cotidiana, un investigador, cuestiona, reflexiona, señala hipótesis, imagina soluciones y las valora antes de poder actuar (Arbeláez et al., 2006).

En un estudio indagatorio histórico para constatar la cantidad de estudiantes que obtuvieron sus títulos profesionales a través de trabajos de investigación, encontramos que solo el $48 \%$ de los estudiantes de las universidades privadas y el $53,4 \%$ de los estudiantes de las universidades públicas obtuvieron su título profesional bajo la modalidad de una tesis (INEI, 2014). Guzmán y Vara (2009), en un estudio realizado sobre las creencias que tienen los docentes sobre el método científico y la influencia que estos tienen en las actitudes del estudiante universitario hacia la investigación, evidenciaron que existen grandes y serias contradicciones además de conflictos entre las posturas didácticas que emplean los docentes en la actividad investigativa, las cuales afectan tanto el interés como las actitudes y el desarrollo competencial que se busca en los estudiantes, condicionándolos o enrumbándolos a elegir los cursos de actualización para obtener su título profesional o grado académico.
Las competencias investigativas propician en los estudiantes de educación superior un aprendizaje significativo que incluye saberes especializados y desarrolla habilidades en la investigación, empleando los resultados que se obtienen para guiar la toma de decisiones, solucionar problemas inmediatos y prospectivos y para construir una teoría que desemboque en acciones innovadoras que retroalimenten su práctica profesional y conducta social (Jaik y Ortega, 2017). En ese sentido, el estudiante debe demostrar el dominio del método científico en la solución de un problema de investigación, empleando para ello todas las etapas que involucra el mismo (Estrada, 2014).

El método científico y la capacidad de la razón nos permiten seguir avanzando en búsqueda de la verdad y en la creación de modelos para aumentar nuestro conocimiento científico del mundo (Asensi-Artiga y ParraPujante, 2002). Los métodos de investigación descritos en la literatura son una herramienta importante para la búsqueda y el perfeccionamiento del conocimiento acerca de la realidad. Sin embargo, es preciso señalar que cada método tiene su forma particular de acercarse al objeto de estudio, lo que origina diversas clasificaciones (Rodríguezy Pérez, 2017). En el transcurso de la historia de la ciencia surgieron diferentes corrientes de pensamiento tales como el materialismo dialéctico, el empirismo, la fenomenología, el positivismo, etc., las mismas que han originado diversos caminos para la búsqueda del conocimiento; sin embargo, en las últimas décadas, dichas corrientes han llegado a polarizarse en dos enfoques principales: enfoque cuantitativo y enfoque cualitativo del método investigativo (Vega-Malagón et al., 2014). En la práctica, estos enfoques son los que polarizan las posturas docentes para la enseñanza-aprendizaje del método científico.

El objetivo del estudio fue el de evidenciar que las posturas didácticas con enfoque cuantitativo vs. conceptualista-epistemológico del docente sobre el método científico influyen en el desarrollo de competencias investigativas en el estudiante universitario, teniendo como objetivos secundarios presentar información descriptiva pertinente al estudio, con el propósito de que se pueda entender mejor los resultados del mismo.

\section{MÉTODOS}

\section{Tipo de estudio}

A continuación de presentan los criterios de la taxonomía de la investigación para clasificar el presente estudio:

Según la intervención del investigador: observacional o sin intervención, porque no se modificó la realidad, ya que esta es únicamente observada. Según el número de variables analíticas: analítica, porque se relacionaron variables. Según la planificación de las mediciones: 
prospectiva, porque en el presente estudio se usaron datos primarios. Según el número de mediciones de la variable de estudio: transversal, porque se utilizó una sola medición en todas las variables. Según el análisis estadístico: el estudio es cuantitativo, porque existió la necesidad de análisis estadístico (Supo y Zacarías, 2020).

\section{Población y muestra}

La población estuvo conformada por 570 estudiantes matriculados en la Universidad de Huánuco (UDH) que cursan su último ciclo de estudios en el primer semestre académico del año 2020; es decir, la población de estudio tiene la característica de estar compuesta por aquellos que están culminando sus estudios universitarios. Según Supo y Zacarías (2020), la población tiene marco muestral conocido, es decir, es considerada como finita. El tamaño de la muestra fue de 215 estudiantes. El tipo de muestreo para la recolección de la muestra corresponde al probabilístico y la técnica de muestreo empleada fue el muestreo estratificado, donde cada carrera profesional es considerada como un estrato.

\section{Instrumentos de recolección de datos}

La técnica de recolección de los datos empleada para analizar la realidad objeto de estudio es la encuesta, con un instrumento de recolección de datos de tipo Likert (con respuestas: muy fácil, fácil, regular, difícil y muy difícil) dirigida a los estudiantes de la Universidad de Huánuco, para que expresen su opinión sobre el desarrollo de sus competencias investigativas y las posturas didácticas de sus docentes respecto al método científico. El instrumento de recolección de datos parte de la escala de evaluación de competencias investigativas (EECl), cuyos autores son Enrique Ortega Rocha y Adla Jaik Dipp. Esta ha sido previamente adaptado y revisada por un juez, el Dr. José Supo Condori, presidente de la Sociedad Hispana de Investigadores Científicos, quien dio la opinión favorable sobre la validez de contenido del instrumento, cuyos ítems estuvieron orientados a obtener información fiable acorde a los objetivos que se persiguen en el estudio.

\section{Procedimientos de la recolección de datos}

El estudio se realizó en el primer semestre del año académico 2020, entre los estudiantes de la Universidad de Huánuco, del último ciclo de estudios. El listado de los estudiantes fue proporcionado por la Oficina de Informática de dicha universidad. Primeramente, se hizo el ajuste del contenido del cuestionario para su posterior validación. A continuación, se llevó a cabo la fase de elección de la muestra de estudiantes. Una vez identificados los sujetos de Investigación fueron administrados los instrumentos de manera virtual, debido al contexto de la pandemia por la covid-19, tiempo en el que se desarrolló la recolección de datos que transcurrió en un tiempo aproximado de diez semanas.

\section{Aspectos éticos}

Se solicitó el permiso correspondiente para acceder al desarrollo del estudio, otorgado por el vicerrectorado académico de la Universidad de Huánuco, para posteriormente solicitar a la Oficina de Informática los datos de los estudiantes, a fin de poder ubicarlos, esencialmente por correo electrónico y complementariamente con el apoyo de sus profesores. La encuesta contiene una introducción que presenta a los estudiantes la naturaleza del estudio e indica el carácter voluntario y anónimo, de su participación, guardando su código universitario, con fines exclusivos para el desarrollo del presente estudio. Al tratarse de un estudio observacional, sin intervención por parte del investigador, no requiere de un pronunciamiento del Comité de Ética en Investigación de la institución.

\section{Análisis de datos}

Al finalizar la fase de recolección de los datos se inició su procesamiento, empleando el software estadístico SPSS versión 24, que facilita la presentación, análisis e interpretación de datos cuantitativos. El análisis de datos se realizó con el procedimiento estadístico chi cuadrado de independencia, el cual es una herramienta estadística no paramétrica, ideal para el análisis de dos variables categóricas.

\section{RESULTADOS}

El análisis de los datos permitió interpretar las respuestas provenientes de la versión de los estudiantes, consiguiendo integrarlas por respuestas obtenidas, por medio de procesos de reflexión con análisis crítico.

Se encontró que aproximadamente dos de cada tres docentes de los estudiantes universitarios que participaron en el estudio tenían una postura didáctica estrictamente cuantitativa $(62,8 \%)$.

Se aprecia un porcentaje considerable de docentes con la postura estrictamente cuantitativa $(62,8 \%)$, de los cuales el $33 \%$ de sus estudiantes adquirieron competencia investigativa. Por el lado de los docentes que tienen una postura conceptualista y epistemológica,

\section{Tabla 1}

Postura didáctica del docente universitario según los estudiantes participantes en el estudio, UDH, 2020-1

\begin{tabular}{lcc}
\hline Postura didáctica & $\mathrm{fi}$ & Porcentaje \\
\hline Estrictamente cuantitativa & 135 & 62,8 \\
Conceptualista y epistemológico & 80 & 37,2 \\
Total & 215 & 100,0 \\
\hline Nota. Información recolectada tras la aplicación de
\end{tabular}

Nota. Información recolectada tras la aplicación de la EECl, UDH, 2020-1. 
Tabla 2

Desarrollo de la competencia investigativa en función de la postura didáctica sobre el método científico, UDH, 2020-1

\begin{tabular}{lccccccc}
\hline & \multicolumn{9}{c}{ Competencia } & & \multicolumn{2}{c}{ Total } \\
Postura & \multicolumn{2}{c}{ No } & \multicolumn{2}{c}{ Si } & & \\
& fi & $\%$ & fi & $\%$ & fi & $\%$ \\
Estrictamente cuantitativa & 64 & 29,8 & 71 & 33,0 & 135 & 62,8 \\
$\begin{array}{l}\text { Conceptualista y } \\
\text { epistemológico }\end{array}$ & 26 & 12,1 & 54 & 25,1 & 80 & 37,2 \\
Total & 90 & 41,9 & 125 & 58,1 & 215 & 100,0 \\
\hline
\end{tabular}

Nota. Información recolectada tras la aplicación de la EECI, UDH, 2020-1

\section{el $25,1 \%$ de sus estudiantes adquirieron competencias} investigativas.

El presente estudio evidencia que las posturas didácticas sobre el método científico con enfoque cuantitativoy conceptualista-epistemológico ciertamente influyen en el desarrollo de las competencias investigativas delestudiante universitario. Esta afirmación se realiza habiéndose obtenido un p-valor de 0,032 (3,2\%), por debajo del nivel de significancia de $5 \%$ considerado en el estudio. El estudio revela que la postura didáctica sobre el método científico que predomina entre los docentes de la Universidad de Huánuco es la estrictamente cuantitativa (62,8 \%). Asimismo, los estudiantes que tienen docentes con esta postura didáctica son los que presentan un mayor porcentaje de competencia investigativa, con respecto a la postura conceptualista-epistemológica.

Guzmán y Vara (2009) encontraron que el dictado de los cursos relacionados a la investigación científica presenta dificultades en los docentes. Mencionan que no es una experiencia uniforme para los estudiantes porque los docentes poseen diversas concepciones sobre el método científico, siendo éstas muchas veces dispares y hasta contradictorias, por lo que estas concepciones afectan la forma cómo se enseña el método científico a los estudiantes. Dicho estudio señala además que el problema no provendría únicamente de la existencia de diversas concepciones sobre el método científico sino también de la discrepancia de criterios que existe entre los docentes y la presión que ellos ejercen sobre los estudiantes para que se acoplen a sus propuestas. Lo anterior, ciertamente menoscaba una enseñanza beneficiosa para el estudiante e incluso puede desmotivar su incursión a la investigación científica.

Los estudiantes que participaron en el estudio fueron, en su mayoría, de la sede principal, lo que estuvo constituido por 9 de cada 10 participantes, aproximadamente. La mayoría de los que participaron en el estudio pertenecen a los programas académicos de Ingeniería Civil, Ingeniería Ambiental, y Derecho y Ciencias Políticas, conformando entre estos el 51,2\% del Total. Se trata principalmente de estudiantes de
Tabla 3

Caracterización de las unidades de estudio participantes, $\mathrm{UDH}, 2020-1$

\begin{tabular}{lcc}
\hline \multirow{2}{*}{ Variable } & $\mathbf{f i}$ & $\%$ \\
\cline { 2 - 3 } & \multicolumn{2}{c}{$\mathbf{n = 2 1 5}$} \\
\hline Ubicación & \multicolumn{2}{c}{} \\
Huánuco & 192 & 89,3 \\
Tingo María & 23 & 10,7 \\
Programa académico & & \\
Ingeniería Civil & 44 & 20,5 \\
Ingeniería Ambiental & 40 & 18,6 \\
Derecho y Ciencias Políticas & 26 & 12,1 \\
Enfermería & 24 & 11,2 \\
Contabilidad y Finanzas & 15 & 7,0 \\
Psicología & 15 & 7,0 \\
Administración de Empresas & 13 & 6,0 \\
Otros & 38 & 17,8
\end{tabular}

Sexo

$\begin{array}{lcc}\text { Masculino } & 81 & 37,7 \\ \text { Femenino } & 134 & 62,3 \\ \text { Situación laboral } & & \\ \text { Estudiante a tiempo completo } & 71 & 33,0 \\ \text { Trabajo a medio tiempo } & 126 & 58,6 \\ \text { Trabajo a tiempo completo } & 18 & 8,4\end{array}$

Nota. Información recolectada tras la aplicación de la EECI, UDH, 2020-1.

sexo femenino; aproximadamente 6 de cada 10 de ellos lo eran. Finalmente, la mayoría de los estudiantes universitarios que participaron en el estudio lo conformaron aquellos que trabajan a medio tiempo; aproximadamente 6 de cada 10 de ellos tenían esta condición. La tercera parte de los estudiantes tenían, no obstante, una dedicación exclusiva a su formación profesional.

\section{DISCUSIÓN}

El presente estudio encontró que la postura didáctica estrictamente cuantitativa y la postura didáctica conceptualista-epistemológica presentan un diferente resultado en cuanto al desarrollo de las competencias investigativas del estudiante universitario, siendo la postura didáctica estrictamente cuantitativa la que genera una mayor eficacia al desarrollar dicha competencia. Sin embargo, es preocupante encontrar que el estudiante se autopercibe con una competencia investigativa limitada o baja, luego de haber aprobado diversos cursos relacionados al método científico, tales como: Estadística, Metodología de la Investigación Científica, Seminario de Tesis 1, Seminario de Tesis $2 \mathrm{y}$, en algunos programas académicos, Seminario de Tesis 3. 
La presencia de contenidos curriculares en el campo de formación en investigación no garantiza el desarrollo de competencias investigativas en los estudiantes universitarios. El $90 \%$ de los estudiantes considera que el programa al que pertenecen debe revaluar la forma en que este orienta la formación investigativa (Pinto y Cortés, 2017). Lo anterior nos indica que cuando el estudiante es consultado, puede aportar ideas y acciones que confirmen las sospechas que se tiene respecto a la forma como se deben hacer las cosas para beneficiar a la colectividad del estudiantado. El estudiante no es ajeno, percibe falencias, las puede comunicar al sentirse escuchado, pero esto debe estar acompañado de acciones holísticas, tanto en la enseñanza como en el aprendizaje.

La enseñanza-aprendizaje del método científico ciertamente es compleja, ya que implica no solamente la participación del docente sino también del estudiante. Cuando el estudiante se encuentra ante una postura didáctica definida por parte del docente, esta debería enriquecer el interés que el estudiante tiene hacia el método científico. Sin embargo, esta postura debe ser lo más holística posible. Los resultados del estudio refieren que los docentes están inclinados por una postura didáctica u otra; existiendo la necesidad de que el estudiante universitario se vea beneficiado por docentes que puedan conocer ampliamente los conceptos epistemológicos del método científico, así como los procedimientos cuantitativos necesarios para el desarrollo de una tesis profesional, es decir, que los docentes adopten una postura híbrida sobre la investigación científica.

En conclusión, con respecto al objetivo principal, se evidencia que las posturas didácticas con enfoque cuantitativo y conceptualista-epistemológico del docente sobre el método científico influyen en el desarrollo de competencias investigativas del estudiante universitario. En base al presente estudio, se concluye que existe evidencia de que las posturas didácticas que emplean los docentes con respecto al método científico influyen en el desarrollo de las competencias investigativas del estudiante universitario. Esta afirmación se hace considerando una probabilidad de error del 3,2\%, la cual está por debajo al nivel de significancia del $5 \%$. El estudio brinda información que indica que la mayoría de los estudiantes universitarios son del sexo femenino, con un rendimiento académico bueno y con la necesidad de laborar a medio tiempo, mientras cursan sus estudios universitarios, con el objeto de afrontar sus gastos personales.

La información recabada en el estudio permite concluir, además, que las posturas didácticas sobre el método científico influyen en el desarrollo de competencias investigativas con un nivel bajo en el estudiante universitario, ya que, considerando una escala vigesimal, se obtuvo una nota promedio de 10,9 en el desarrollo de dichas competencias, siendo la postura didáctica estrictamente cuantitativa la que brinda una mayor proporción de estudiantes con competencias investigativas. Del mismo modo, el estudio brinda información que permite concluir que aproximadamente 6 de cada 10 estudiantes consideran que poseen competencias investigativas para afrontar su tesis profesional, siendo éstas, no obstante, bajas o limitadas.

\section{REFERENCIAS}

Abreu, O., Gallegos, M., Jácome, J. y Martínez, R. (2017). La Didáctica: Epistemología y Definición en la Facultad de Ciencias Administrativas y Económicas de la Universidad Técnica del Norte del Ecuador. Formación universitaria, 10(3), 81-92. https://scielo.conicyt.cl/scielo.php?script=sci_abstract\&pi$\mathrm{d}=$ S0718-50062017000300009\&lng=es\&nrm=iso

Arbeláez, R., Hernández, N. y Pérez, M. (2006). El desarrollo de la competencia investigadora en los estudiantes de pregrado. Docencia Universitaria, 7(1), 5-10. https://revistas.uis.edu.co/index.php/revistadocencia/article/view/769/1073

Asensi-Artiga, V. y Parra-Pujante, A. (2002). El método científico y la nueva filosofía de la ciencia. Anales de Documentación, 5, 9-19. https://revistas.um.es/ analesdoc/article/view/2251

Estrada, O. (2014). Sistematización teórica sobre la competencia investigativa. Revista Electrónica Educare, 18(2), 177-194. https://dialnet.unirioja.es/servlet/ articulo?codigo $=4780914$

Guzmán, C., y Vara, A. (2009). Creencias docentes sobre el método científico y su influencia en las actitudes hacia la investigación y la disposición para realizar tesis en una universidad privada de Lima. Revista Cultura, 21, 341'371. http://www.revistacultura.com.pe/revistas/RCU_21_1_creencias-docentes-sobre-el-metodo-cientifico-y-su-influencia-en-las-actitudes-hacia-la-investigacion-y-la-disposicion-para-realizar-tesis-en-una-universidad-privada-de-lima.pdf

Instituto Nacional de Estadística e Informática, INEI. (2014). Encuesta Nacional a Egresados Universitarios y Universidades. https://www.inei.gob.pe/media/MenuRecursivo/publicaciones_digitales/Est/Lib1298/ Libro.pdf

Jaik, A. y Ortega, E. (2017). Validación de la escala para evaluar Competencias Metodológicas de Investigación [conferencia]. XIV Congreso Nacional de Investigación Educativa, San Luis Potosí, México. https:// www.comie.org.mx/congreso/memoriaelectronica/ v14/doc/2790.pdf

Orozco, V. (2013). Los criterios epistemológicos en la investigación económica. Pensamiento critico, 18(1), 63-79. revistasinvestigacion.unmsm.edu.pe/index. php/econo/article/download/8916/7744

Pinto, A. y Cortés, O. (2017). ¿Qué piensan los estudiantes universitarios frente a la formación investigativa? REDU: Revista de Docencia Universitaria, 15(2), 57 76. https://n9.cl/ikz10 
Rodríguez, A. y Pérez, A. (2017). Métodos científicos de indagación y de construcción del conocimiento. Revista EAN, 82, 179-200. https://doi. org/10.21158/01208160.n82.2017.1647

Salazar, D. (2004). Didáctica, Interdisciplinariedad y trabajo científico en la formación del profesor. Editorial Pueblo y Educación. https://n9.cl/qd025

Supo, J. y Zacarías, H. (2020). Metodología de la Investigación Científica: Para Las Ciencias de la Salud y Las Ciencias Sociales (3a ed.). Independently Published. https://books.google.com.pe/books?i$d=$ WruXzQEACAAJ

Vega-Malagón, G., Ávila-Morales, J., Vega-Malagón, A., Camacho-Calderón, N., Becerril-Santos, A. y Leo-Amador, G. (2014). Paradigmas en la investigación. Enfoque cuantitativo y cualitativo. 10(15), 6. https:// n9. $\mathrm{cl} / \mathrm{t} 5 \mathrm{qOa}$

\section{Contribución de los autores}

HRZV: conceptualización, metodología, recursos, recolección de datos, visualización, análisis formal, análisis de resultados, discusión y revisión final del artículo.

JVG: metodología, supervisión, visualización, redacción, revisión y edición.

JASC: metodología, análisis formal, validación, redacción, revisión y edición.

\section{Fuentes de financiamiento}

La investigación fue realizada con recursos propios.

\section{Conflictos de interés}

Los autores declaran no tener conflicto de interés.

\section{Correspondencia}

Héctor R. Zacarías Ventura

Dirección: Calle Juan Blásquez 136, Huánuco, Perú

CP.: 100101

Cel.: 976674911

Email: Hector.Zacarias@udh.edu.pe 